\title{
Tractable field metrics for restoration and recovery monitoring of staghorn coral Acropora cervicornis
}

\author{
C. Kiel $^{1}$, B. E. Huntington ${ }^{2}$, M. W. Miller ${ }^{2, *}$ \\ ${ }^{1}$ Division of Marine Affairs, Rosenstiel School of Marine and Atmospheric Sciences, University of Miami, Miami, \\ Florida 33149, USA \\ ${ }^{2}$ National Marine Fisheries Service, Southeast Fisheries Science Center, Miami, Florida 33149, USA
}

\begin{abstract}
The staghorn coral Acropora cervicornis is a branching coral with complex morphology. While this structural complexity confers high ecological and habitat value, it hinders accurate quantification. An appropriate and quantitative coral 'unit' is required to effectively implement protective provisions (e.g. prevention of take or strategies to meet quantitative recovery criteria) for A. cervicornis under the United States Endangered Species Act. Actual measurements of 3dimensional structure or extent of branches within colonies are time-consuming and therefore not feasible for monitoring application. This study calibrated arduous in-water measurements of total linear extent (TLE, sum of all branch lengths in a fully live colony, intuitively representing the 'amount' of coral in an individual colony) to more manageable standard field measurements of colony dimensions or photographs. The robustness of this relationship was then evaluated among colonies of different origin (wild, nursery-reared, transplanted) and different habitats (patch reef, shallow forereef, deep forereef) in the upper Florida Keys, USA. Within this sample set, colony volume (calculated as an ellipsoid based on 3 colony dimensions) was highly predictive of TLE $\left(\mathrm{r}^{2}=\right.$ 0.94 for log-log linear regression), and this relationship was consistent for colonies from different origins and different reef habitats. Thus, 'unit' coral abundance at the colony scale in terms of branch extent (TLE) can be reliably estimated from simple colony dimensions in the Florida Keys. Additional data are required to determine if different calibrations are needed in other populations, to adjust estimates for partial mortality of colonies, and to 'scale up' reliable estimates to thicketor reef-scale.
\end{abstract}

KEY WORDS: Florida Keys $\cdot$ Abundance $\cdot$ Apical tips $\cdot$ Methodology

\section{INTRODUCTION}

Corals are declining in the Caribbean due to natural and anthropogenic stressors. Some species such as Acropora cervicornis, commonly known as staghorn coral, are considered at risk of extinction, as reflected by its categorization as 'Critically Endangered' under the IUCN Red List (NMFS 2006, Carpenter et al. 2008). This is alarming because this species provides architectural structure that supports numerous other species in reef ecosystems. Additionally, staghorn coral contributes directly to ecological and economic services, including reef growth, island formation, coastal protection, fisheries habitat, biodiversity, and even nutrient cycling (Bruckner 2003).

In 2006, Acropora cervicornis was listed as a threatened species under the United States Endangered Species Act (ESA). The ESA provides legal mandates to 'recover' listed species populations to meet quantitative recovery criteria (when legal protections would no longer be required for species sustainability) and to protect their critical habitats with the goal of conserving the ecosystems upon which listed species depend. Many components of this statute, including requirements for ongoing status review, protections against 'take' of the species, mit- 
igation for unavoidable actions that involve 'take' (e.g. dredging projects), or evaluation of particular recovery actions all require an appropriate 'unit' definition for listed species as a standardized means of quantifying loss, abundance, or progress toward recovery. Standard units such as number of individuals or biomass (used for other ESA listed species) are elusive in colonial branching corals. The complex branching morphology of A. cervicornis colonies makes standard coral demographic metrics such as colony diameter or percent live cover (e.g. Bak \& Meesters 1998) less informative and more difficult to assess for this species. Recent efforts to improve quantification of coral abundance in situ range from intensive computer models of actual surface area (Bythell et al. 2001, Courtney et al. 2007) to various geometric surrogates for non-branching species (e.g. hemispherical to box-shaped; Fisher et al. 2007). Yet, these approaches are time-consuming (Bythell et al. 2001), have high errors when applied to branching species (Courtney et al. 2007), and have not been developed specifically for A. cervicornis. Therefore, defensible, repeatable metrics that can be efficiently quantified are needed to determine the abundance of A. cervicornis to effectively implement the protective provisions of the ESA.

Active restoration has already been employed to address the depleted state of Acropora cervicornis in the Caribbean Sea (Young et al. 2012). For example, in the Florida Keys, multiple genets of A. cervicornis are actively cultured in field nurseries and have been outplanted to a variety of reefs over the past $5 \mathrm{yr}$ (e.g. Coral Restoration Foundation; www.coralrestoration. org) with the goal of restoring small, genetically diverse populations that can serve as larval sources to seed surrounding reefs. Total linear extent (TLE, the sum of branch lengths within an entire colony; Fig. 1A) is a metric used in these coral nurseries to track production of A. cervicornis (Johnson et al. 2011) and provides an intuitive representation of a unit or amount of branching coral. However, TLE measurements are time-consuming even on small colonies and rapidly become impractical as colonies become larger.

Acropora cervicornis colonies grow by extension and branching of apical branch tips. Hence, the abundance of apical tips within a colony (Fig. 1) gives an indicator of the potential productivity of that colony, which can be estimated by combining the branching rate (how often an apical tip branches into 2 or more apical tips) with the mean rate of linear extension for each branch (e.g. Shinn 1976). As with measuring TLE, counting the number of apical tips is a time-consuming process, especially among larger colonies. A rapid, reliable means to estimate the number of apical tips would allow efficient estimation of growth potential of a given colony.

The goal of the present study was to streamline in situ measurements of Acropora cervicornis colonies to enable a defensible 'unit' abundance estimate. We tested whether TLE could be reliably approximated

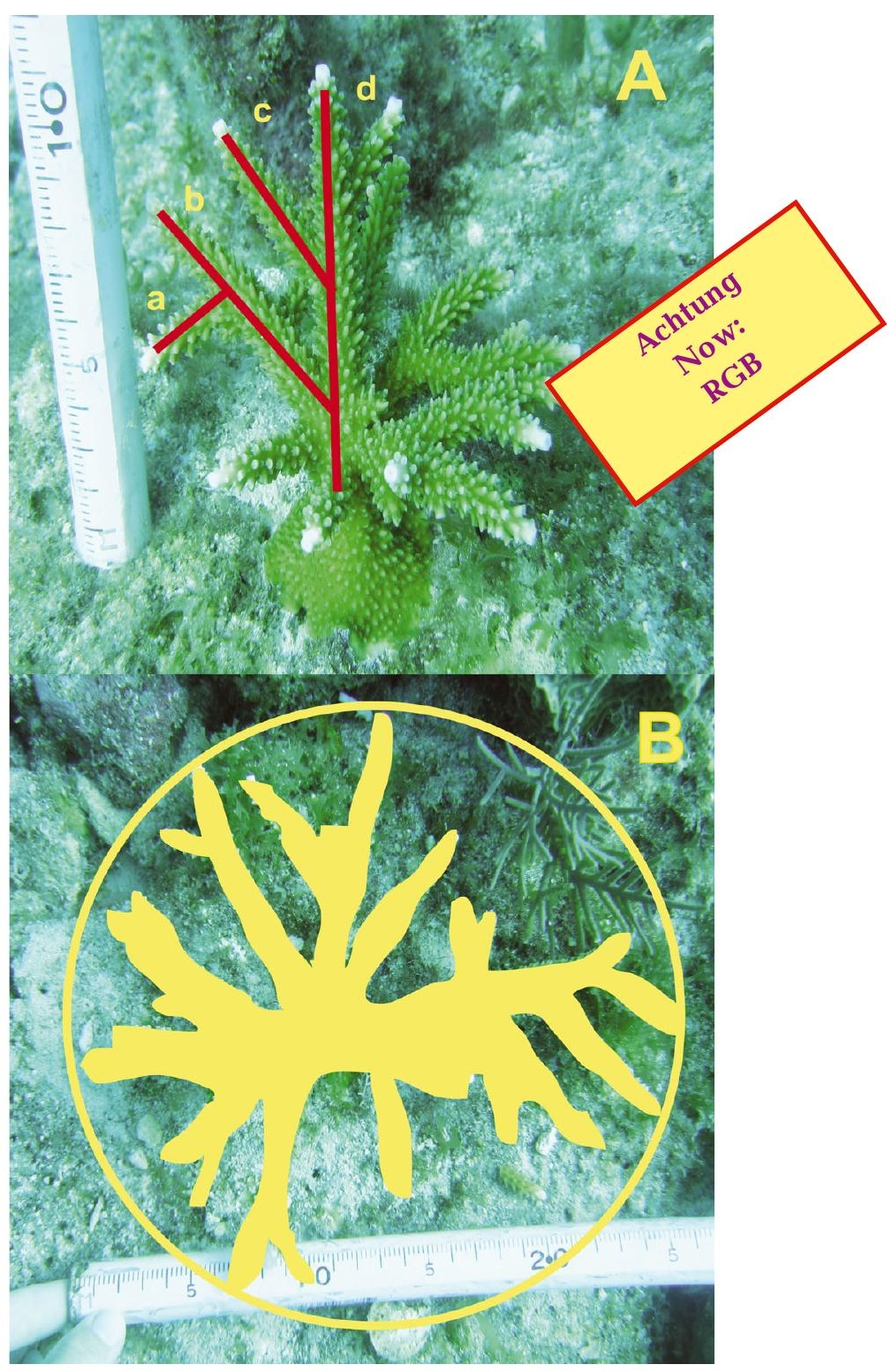

Fig. 1. Acropora cervicornis. Illustration of colony metrics. (A) The red stick figure depicts the measurements summed for linear extent for a branch of a colony. All branches in the colony were summed to get the total linear extent of the colony, the most intuitive measure of 'total amount of coral'. Approximately 14 apical tips are visible on this colony. (B) Projected area of a colony which was manually outlined on the scaled, top-down colony photographs 
from (1) field measurements of straightforward colony dimensions (length, width, height) and/or (2) a 2dimensional projected colony area (i.e. footprint) over a range of colony sizes in the upper Florida Keys. Next, we evaluated the robustness of such estimates among colonies of varying origin (e.g. restored versus wild colonies) or habitat (e.g. patch reef, shallow forereef, deep forereef). Lastly, we explored the possibility of using these same colony dimensions to estimate the number of apical tips on a colony as a proxy for colony growth potential.

\section{MATERIALS AND METHODS}

\section{Study sites and field measurements}

Live colonies of Acropora cervicornis have been transplanted at numerous sites along the Florida Reef Track since 2008 for restoration and experimental purposes. Colonies originating from culture in field nurseries (K. Nedimyer pers. comm.), experimentally transplanted wild colonies (M. Miller unpubl. data), and unmanipulated wild colonies were measured and compared in the current study. Outplanted nursery colonies were measured at 6 different sites, transplanted wild colonies were measured at 2 of these 6 sites, and unmanipulated wild colonies were measured at 2 additional sites between May 2011 and January 2012 (Table 1). These sites also represent contrasting reef geomorphology or habitat types, including mid-shelf patch reefs, shallow forereef, and deeper forereef (Table 1). These populations are all sparse, with individual colonies readily distinguishable.

A total of 79 fully live colonies (i.e. negligible partial mortality) were chosen haphazardly to represent the range of colony sizes present (actual range: 6 to $1910 \mathrm{~cm}$ TLE or 12 to $70 \mathrm{~cm}$ diameter). For each selected colony, the following dimensions were measured (all in $\mathrm{cm}$ ): maximum colony diameter (length, L), perpendicular to maximum diameter (width, $W$ ), maximum colony height $(H)$ above the benthos, and TLE (Fig 1A) of branches were measured. The total number of apical tips for each colony was counted. A top-down, scaled digital photograph was taken of each colony. A meter $\operatorname{rod}(L, W, H)$ or flexible meter tape (TLE) was used for colony measurements. Three colonies were re-measured during the same dive by the same observer to estimate repeatability of the in situ measurements. Duplicate TLE measurements differed by $<7.4 \%$, suggesting minimal sampling error in the colony measurements.

Once branching corals grow larger than about $20 \mathrm{~cm}$ TLE, their overall shape resembles an ellipse (C. Kiel pers. obs.). Therefore, $L, W$, and $H$ were used to calculate an estimated ellipsoid volume (EV):

$$
\mathrm{EV}=(4 / 3) \times \pi \times H / 2 \times L / 2 \times W / 2
$$

To calculate the projected (2-dimensional) area of the colonies, digital photographs were first scaled using the calibration tool in Coral Point Count with Excel extensions (Kohler \& Gill 2006) to the scale bar in the image. The outline of the live coral branches was then traced as closely as possible, and the outlined area was calculated (Fig. 1B). Only 42 colonies were included in this analysis, since the scale was not appropriately placed in some photographs, precluding areal calibration for those colonies.

Data sets were checked for normality using ShapiroWilk's tests, and were transformed $\left(\log _{10}\right)$ for parameters where required to meet assumptions of normality (TLE, EV, number of apicals). Simple linear regressions were run between the TLE (as the independent variable), and the derived parameters of EV and projected area as single dependent variables. The relationship between number of apicals (independent variable), and the derived parameter of EV was also explored through simple linear regression. Lastly, 2 ANCOVAs were performed on the same TLE $\times$ EV

Table 1. Site name, habitat type, coordinates, and depth of study sites, all located in the upper Florida Keys

\begin{tabular}{|lcccc|}
\hline Site & Habitat type & Coordinates & Depth $(\mathrm{m})$ & Colony origin \\
\hline Aquarius & Deep forereef & $24^{\circ} 57.010^{\prime} \mathrm{N}, 80^{\circ} 27.130^{\prime} \mathrm{W}$ & 18 & Nursery and transplant \\
Conch Shallow & Shallow forereef & $24^{\circ} 57.083^{\prime} \mathrm{N}, 80^{\circ} 27.594^{\prime} \mathrm{W}$ & 5 & Nursery and transplant \\
Pickles & Shallow forereef & $24^{\circ} 59.170^{\prime} \mathrm{N}, 80^{\circ} 24.940^{\prime} \mathrm{W}$ & 9 & Nursery \\
Molasses & Shallow forereef & $25^{\circ} 00.740^{\prime} \mathrm{N}, 80^{\circ} 22.400^{\prime} \mathrm{W}$ & 9 & Nursery \\
French & Shallow forereef & $25^{\circ} 02.057^{\prime} \mathrm{N}, 80^{\circ} 20.893^{\prime} \mathrm{W}$ & 9 & Nursery \\
Key Largo Dry Rocks & Patch reef & $25^{\circ} 07.200^{\prime} \mathrm{N}, 80^{\circ} 18.000^{\prime} \mathrm{W}$ & 6 & Nursery \\
Tavernier Patch A & Patch reef & $24^{\circ} 59.228^{\prime} \mathrm{N}, 80^{\circ} 27.173^{\prime} \mathrm{W}$ & 5 & Wild \\
Tavernier Patch B & Patch reef & $24^{\circ} 59.242^{\prime} \mathrm{N}, 80^{\circ} 27.159^{\prime} \mathrm{W}$ & 5 & Wild \\
Little Conch & Patch reef & $24^{\circ} 56.550^{\prime} \mathrm{N}, 80^{\circ} 28.430^{\prime} \mathrm{W}$ & 5 & Wild \\
\hline
\end{tabular}


data to assess homogeneity of slopes for colonies from the 3 different habitat types and for colonies of the 3 different origins. A reduced data set $(\mathrm{n}=63$ for colony origin; $\mathrm{n}=63$ for habitat) was used for each ANCOVA to ensure a similar TLE range was represented in each of the subgroups.

\section{RESULTS}

EV (based on field-measured colony dimensions) was a significant predictor of the total amount of coral (TLE) for all colonies (Fig. 2, Table 2; $\mathrm{R}^{2}=$ $0.94, p<0.001$ ). When colonies were categorized by differing habitat types (Fig. 2B) or differing origins (Fig. 2C), ANCOVA indicated no significant difference in the slopes calculated for each group (Table 2). Hence, the overall regression relationship (Table 3 ) is robust amongst the habitats and colony origins within this data set. The projected colony area calculated from the scaled photographs also provided a strong correlation for TLE (Fig. 3; $\mathrm{R}^{2}=0.81, \mathrm{p}<$ 0.001 ), with a smaller data set of 42 colonies that had adequately scaled photos. EV was also a significant predictor of the number of apical tips on a colony (Fig. 4, Table 3; $\mathrm{R}^{2}=0.73, \mathrm{p}<0.001$ ).

\section{DISCUSSION}

It is possible to streamline and calibrate tractable in situ measurements of Acropora cervicornis colony dimensions that accurately estimate arduous timeconsuming measurements of colony branch extent (TLE, i.e. complex habitat or unit 'amount' of coral) or number of apical tips based on a sample population in the upper Florida Keys. This population included both transplanted (including nursery-reared colonies) and wild in situ colonies, and spanned midshore patch reef $(<7 \mathrm{~m}$ depth), shallow forereef $(\sim 7 \mathrm{~m})$, and deeper forereef (15 to $18 \mathrm{~m}$ depth) habitats. The predictive relationship was robust across these categories of habitat and colony origin (Table 2). Therefore, this study provides a protocol for estimating colony TLE, representing a true unit of coral as well as a proxy for the 3-dimensional structure of the colony, from 3 simple dimensional in situ measurements of length, width, and height. TLE is currently used as a performance measure in field nurseries and in evaluating early stages of nursery outplants (Johnson et al. 2011), but is time-consuming even for small colonies and soon becomes impracticable as colonies grow larger. Results reported here show that colonies
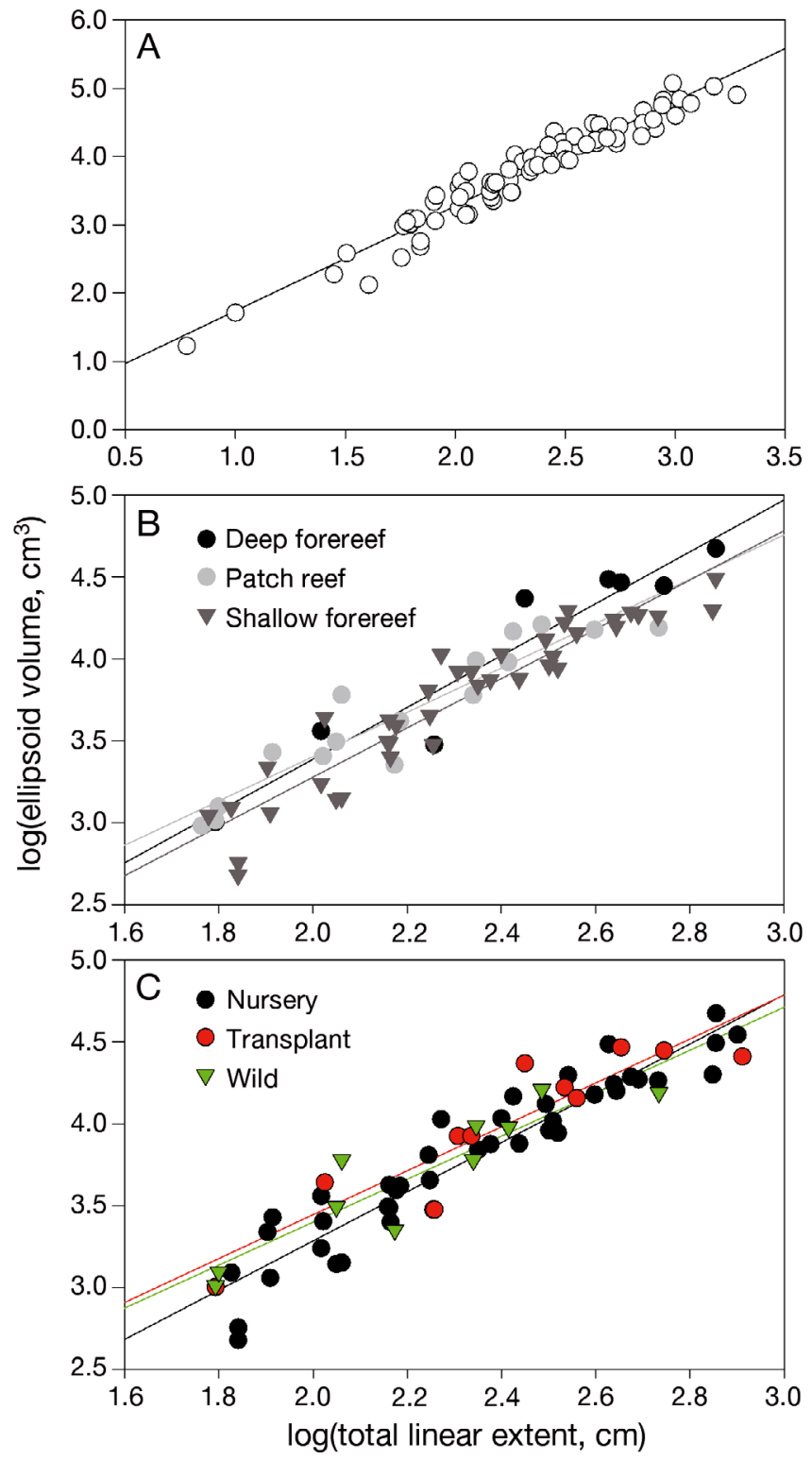

Fig. 2. Acropora cervicornis. (A) Relationship between total linear extent measured in the field and ellipsoid volume of the colony calculated from 3 colony dimensions $\left(n=79, R^{2}=\right.$ $0.94, p<0.001)$. Other panels show the same relationship parsed by (B) colonies from different habitat types or (C) different origins. ANCOVA indicates that these slopes are not significantly different so the relationship shown in (A) is robust to these factors. Statistical results are given in Table 2, and regression parameters in Table 3

restocked from field nursery culture resembled wild in situ colonies in their colony morphology and, hence, the same regression metrics could be used. Similarly, colonies growing in different habitat types (including depths from 5 to $18 \mathrm{~m}$ ) were shown not to differ significantly in morphological relationships. This is somewhat surprising given previous descriptions of 
Table 2. Acropora cervicornis. Regression results of the relationship between estimated volume (EV) and total linear extent (TLE) for staghorn coral colonies: simple linear regression results for the full data set; ANCOVA results from the restricted data sets evaluating the relationship between EV and TLE among A. cervicornis colonies in different reef habitat types and of differing origin

\begin{tabular}{|lccccc|}
\hline Source & df & SS & F-ratio & $p$ & $R^{2}$ \\
\hline Full data set $(\mathbf{n}=\mathbf{7 9})$ & & & & & \\
Model & 1 & 41.24 & 1173.80 & $<0.001$ & 0.94 \\
Error & 77 & 2.71 & & & \\
Differing habitat type $(\mathbf{n}=\mathbf{6 3})$ & & & & & \\
$\log ($ TLE) & 1 & 9.51 & 358.10 & $<0.001$ & \\
Reef habitat & 2 & 0.15 & 2.73 & 0.074 & \\
Reef habitat $\times \log ($ TLE) & 2 & 0.03 & 0.63 & 0.535 & \\
Differing colony origin $(\mathbf{n}=\mathbf{6 3})$ & & & & & \\
$\log ($ TLE) & 1 & 7.07 & 239.88 & $<0.001$ & \\
Colony origin & 2 & 0.10 & 1.71 & 0.190 & \\
Colony origin $\times \log ($ TLE) & 2 & 0.04 & 0.67 & 0.518 & \\
\hline
\end{tabular}

differing colony growth and branching patterns with depth (e.g. Tunnicliffe 1983). Unfortunately, all the deep colonies included in the present study were transplanted or nursery-raised as no wild colonies were encountered in deep habitats in the upper Florida Keys, so caution should be used in generalizing this finding to deep wild colonies.

Additional caliper measurements of branch diameter could be employed to calculate colony surface area (as a cylindrical surface area based on branch diameter and length indicated by TLE) to estimate habitat provision as well as other functional attributes such as fecundity, respiration, produc-

Table 3. Acropora cervicornis. Predictive regression relationships between simple estimates of staghorn colony volume $\left(\mathrm{EV}, \mathrm{cm}^{3}\right)$ or colony area $\left(\mathrm{cm}^{2}\right)$ to various morphological parameters of interest. Two-dimensional colony area derived from top-down colony photographs

Equation corrected after publication

\begin{tabular}{|ccc|}
\hline Parameter $(x)$ & Equation & Fit $\left(R^{2}\right)$ \\
\hline $\begin{array}{c}\text { Total linear } \\
\text { extent }(\mathrm{EV})\end{array}$ & $10\left[\frac{\log _{10}(\mathrm{EV})-0.203}{1.538}\right]$ & 0.94 \\
$\begin{array}{c}\text { Total linear } \\
\text { extent (area) }\end{array}$ & $10\left[\frac{\log _{10}(\text { colony area })+0.161}{1.174}\right]$ & 0.81 \\
$\begin{array}{c}\text { Number of } \\
\text { apical tips }\end{array}$ & $10\left[\frac{\log _{10}(\mathrm{EV})+1.089}{1.748}\right]$ & 0.73 \\
\hline
\end{tabular}

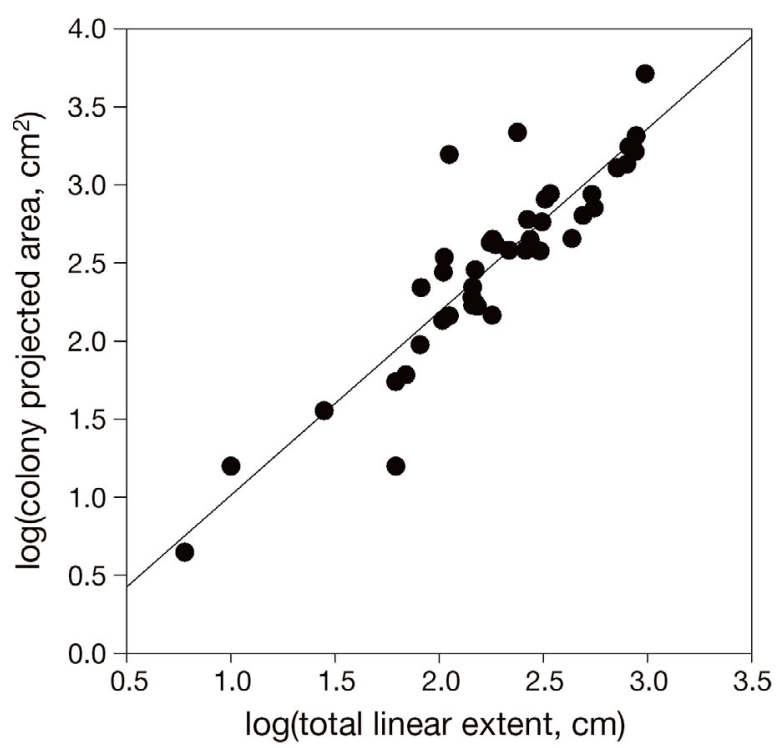

Fig. 3. Acropora cervicornis. Relationship of total linear extent measured in the field to projected colony area $(n=42$, $\mathrm{R}^{2}=0.81, \mathrm{p}<0.001$ ) derived from scaled photographs of individual colonies. All regression parameters are given in Table 3 tivity, etc. It should be noted that we selected fully live colonies in this study. It should be feasible to scale the TLE estimates derived from the equations presented here to visual estimates of partial mortality at the colony scale. Additional data should validate this approach.

Results given here are based on log-transformed data to meet statistical assumption requirements. Hence, the predictive relationship between colony dimensions and TLE has larger error among larger colony sizes. Human error in our TLE measurements was also likely larger in bigger colonies. Additional measurements of larger colonies will help resolve

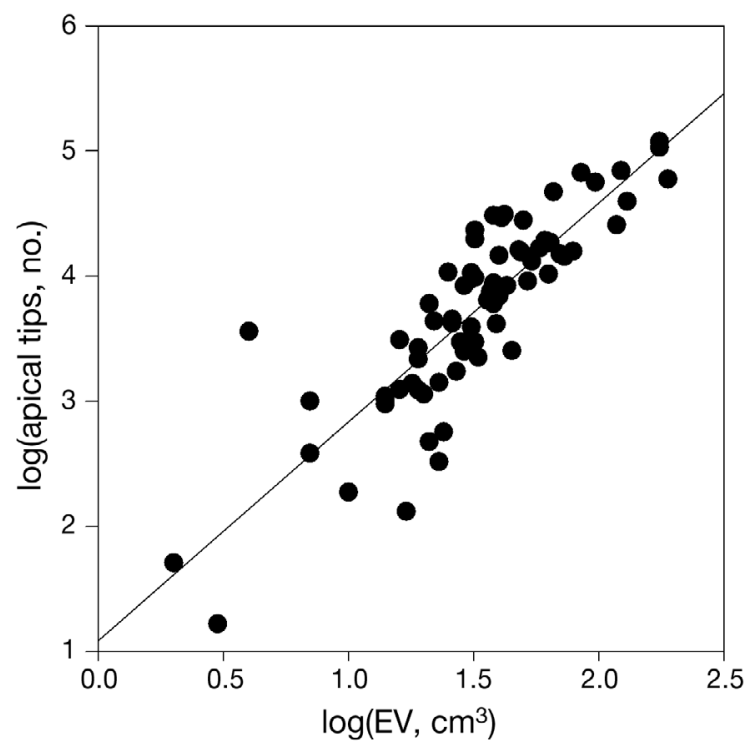

Fig. 4. Acropora cervicornis. Relationships between number of apical tips measured in the field and ellipsoid volume (EV) of the colony calculated from 3 colony measurements $\left(\mathrm{n}=73, \mathrm{R}^{2}=0.73, \mathrm{p}<0.001\right)$. All regression parameters are given in Table 3 
this. The current study also sampled individual Acropora cervicornis colonies with readily discernible boundaries; thickets of A. cervicornis are largely absent in the upper Florida Keys study area. Additional work is also needed to develop metrics at the meso-scale where individual A. cervicornis colonies merge into thickets. Current metrics for thickets include GPS mapping of thicket extent, with or without incorporating estimates of percent live cover within the thicket (e.g. Devine et al. 2005, Walker et al. 2012), although the geographic migration and 'spreading' of a thicket can yield ambiguity as to whether the amount of coral within that thicket is increasing, decreasing, or remaining stable (Walker et al. 2012). Because the status/abundance of thickets is an important criterion for recovery of the species and its full ecological functioning, improved means of quantification for thickets are needed, and scalingup the colony-scale metrics presented here may be important for monitoring the recovery of this species.

Additional data are also needed to validate or supplement the predicted equations for colonies in different geographic regions and additional habitats, but the consistency for colonies in the upper Florida Keys from different origins and across habitats suggests a high potential for the transferability of these results.

Acknowledgements. This work was made possible by funding from NOAA's Coral Reef Conservation Program, NMFS Southeast Fisheries Science Center, and NMFS Southeast Regional Office. Research/field sampling was conducted under Florida Keys National Marine Sanctuary Permit No. FKNMS-2011-032 to M.W.M. Logistical support from the University of North Carolina Wilmington-Aquarius Reef Base and field assistance by K. Lohr are gratefully acknowledged.

\section{LITERATURE CITED}

Bak RPM, Meesters EH (1998) Coral population structure: the hidden information of colony size-frequency distribu-

Editorial responsibility: Paul Snelgrove, St. John's, Newfoundland and Labrador, Canada tions. Mar Ecol Prog Ser 162:301-306

Bruckner AW (2003) Proceedings of the Caribbean Acropora workshop: potential application of the U.S. Endangered Species Act as a conservation strategy. Tech Memo NMFS-OPR-24, NOAA, Silver Spring, MD

Bythell J, Pan P, Lee J (2001) Three-dimensional morphometric measurements of reef corals using underwater photogrammetry techniques. Coral Reefs 20:193-199

Carpenter KE, Abrar M, Aeby G, Aronson RB and others (2008) One-third of reef-building corals face elevated extinction risk from climate change and local impacts. Science 321:560-563

Courtney LA, Fischer WS, Raimondo S, Oliver LM, Davis WP (2007) Estimating three-dimensional colony surface area of field corals. J Exp Mar Biol Ecol 351:234-242

Devine B, Rogers CS, Loomis C (2005) Mapping marine populations: using surface water GPS for spatial analysis. Proc Gulf Caribb Fish Inst 56:411-420

Fisher WS, Davis W, Quarles R, Patrick J and others (2007) Characterizing coral condition using estimates of threedimensional colony surface area. Environ Monit Assess 125:347-360

Johnson ME, Lustic C, Bartels E, Baums IB and others (2011) Caribbean Acropora restoration guide: best practices for propagation and population enhancement. The Nature Conservancy, Arlington, VA

- Kohler KE, Gill SM (2006) Coral Point Count with Excel extensions (CPCe): a Visual Basic program for the determination of coral and substrate coverage using random point count methodology. Comput Geosci 32:1259-1269

NMFS (National Marine Fisheries Service)(2006) Endangered and threatened species: final listing determinations for elkhorn coral and staghorn coral. Fed Regist 71: 26852-26861

Shinn EA (1976) Coral reef recovery in Florida and the Persian Gulf. Environ Geol 1:241-254

Tunnicliffe VJ (1983) Caribbean staghorn coral populations: pre-hurricane Allen conditions in Discovery Bay, Jamaica. Bull Mar Sci 33:132-151

> Walker BK, Larson EA, Moulding AL, Gilliam DS (2012) Small-scale mapping of indeterminate arborescent acroporid coral (Acropora cervicornis) patches. Coral Reefs 31:885-894

Young CN, Schopmeyer SA, Lirman D (2012) A review of reef restoration and coral propagation using the threatened genus Acropora in the Caribbean and western Atlantic. Bull Mar Sci 88:1075-1098

Submitted: June 12, 2012; Accepted: October 31, 2012

Proofs received from author(s): December 13, 2012 